APPEARED IN BULLETIN OF THE

AMERICAN MATHEMATICAL SOCIETY

Volume 26, Number 2, April 1992, Pages 269-275

\title{
NILPOTENT ORBITS, NORMALITY, AND HAMILTONIAN GROUP ACTIONS
}

\author{
RANeE Brylinski and Bertram Kostant
}

\begin{abstract}
Let $M$ be a $G$-covering of a nilpotent orbit in $\mathfrak{g}$ where $G$ is a complex semisimple Lie group and $\mathfrak{g}=\operatorname{Lie}(G)$. We prove that under Poisson bracket the space $R[2]$ of homogeneous functions on $M$ of degree 2 is the unique maximal semisimple Lie subalgebra of $R=R(M)$ containing $\mathfrak{g}$. The action of $\mathfrak{g}^{\prime} \simeq R[2]$ exponentiates to an action of the corresponding Lie group $G^{\prime}$ on a $G^{\prime}$-cover $M^{\prime}$ of a nilpotent orbit in $\mathfrak{g}^{\prime}$ such that $M$ is open dense in $M^{\prime}$. We determine all such pairs $\left(\mathfrak{g} \subset \mathfrak{g}^{\prime}\right)$.
\end{abstract}

The theory of coadjoint orbits of Lie groups is central to a number of areas in mathematics. A list of such areas would include (1) group representation theory, (2) symmetry-related Hamiltonian mechanics and attendant physical theories, (3) symplectic geometry, (4) moment maps, and (5) geometric quantization. From many points of view the most interesting cases arise when the group $G$ in question is semisimple. For semisimple $G$ the most familar of the orbits are of semisimple elements. In that case the associated representation theory is pretty much understood (Borel-Weil-Bott and noncompact analogues, e.g., Zuckerman functors). The isotropy subgroups are reductive and the orbits are in one form or another related to flag manifolds and their natural generalizations.

A totally different experience is encountered with nilpotent orbits of semisimple groups. Here the associated representation theory (unipotent representations) is poorly understood and there is a loss of reductivity of isotropy subgroups. To make matters worse (or really more interesting) orbits are no longer closed and there can be a failure of normality for orbit closures. In addition simple connectivity is generally gone but more seriously there may exist no invariant polarizations.

The interest in nilpotent orbits of semisimple Lie groups has increased sharply over the last two decades. This perhaps may be attributed to the reoccuring experience that sophisticated aspects of semisimple group theory often leads one to these orbits (e.g., the Springer correspondence with representations of the Weyl group).

In this note we announce new results concerning the symplectic and algebraic geometry of the nilpotent orbits $O$ and the symmetry groups of that geometry. The starting point is the recognition (made also by others) that the ring $R$ of regular functions on any $G$-cover $M$ of $O$ is not only a Poisson algebra (the case for any coadjoint orbit) but that $R$ is also naturally graded. The key theme is that the

1991 Mathematics Subject Classification. Primary 22E46; Secondary 58F05, 58F06, 32M05, 14L30.

Received by the editors March 29, 1991 and, in revised form, May 29, 1991

The first author is an Alfred P. Sloan Fellow

The work of the second author was supported in part by NSF Grant DMS-8703278 
same nilpotent orbit may be "shared" by more than one simple group, and the key result is the determination of all pairs of simple Lie groups having a shared nilpotent orbit. Furthermore there is then a unique maximal such group and this group is encoded in the symplectic and algebraic geometry of the orbit. Remarkably a covering of nilpotent orbit of a classical group may "see" an exceptional Lie group as the maximal symmetry group of this sympectic manifold. A beautiful instance of this is that $G_{2}$ is the symmetry group of the simply connected covering of the maximal nilpotent orbit of $\operatorname{SL}(3, \mathbb{C})$ and that this six-dimensional space "becomes" the minimal nilpotent orbit of $G_{2}$.

Our work began with a desire to thoroughly investigate a striking discovery of Levasseur, Smith, and Vogan. They found that the failure of the closure of the eight-dimensional nilpotent orbit of $G_{2}$ to be a normal variety may be "remedied" by refinding this orbit as the minimal nilpotent orbit of $\operatorname{SO}(7, \mathbb{C})$. The failure has a lot to do with the seven-dimensional representation of $G_{2}$. In general given $M$ we have found that there exists a unique minimal representation $\pi$ (containing the adjoint) wherein $M$ may be embedded with normal closure. It was the study of $\pi$ that led to the discovery of the maximal symmetry group $G^{\prime}$. Using a new general transitivity result for coadjoint orbits we prove that, modulo a possible normal Heisenberg subgroup (and that occurs in only one case), $G^{\prime}$ is semisimple.

Past experience has shown that the action of a subgroup $H$ on a coadjoint orbit of $G$ is a strong prognostigator as to how the corresponding representation $L$ of $G$ decomposes under $H$. If this continues to hold for unipotent representations our classification result should yield all cases where $L$ remains irreducible (or decomposes finitely) under a semisimple subgroup.

\section{ThE MAXIMAL SYMMETRY GROUP AND "SHARED" ORBITS}

Let $G$ be a simply connected complex semisimple Lie group and $\mathfrak{g}$ the Lie algebra of $G$. Let $e \in \mathfrak{g}$ be nilpotent and assume (for simplicity of exposition but with no real loss) that $e$ has nonzero projection in every simple component of $\mathfrak{g}$. Let $O$ be the adjoint orbit of $e$ and let $\nu: M \longrightarrow O$ be a $G$-covering. Let $R=R(M)$ be the ring of regular functions on $M$ and let $R[\mathfrak{g}]$ be the copy of $\mathfrak{g}$ in $R$ defining the moment map $M \longrightarrow \mathfrak{g}^{*}$. Identify $\mathfrak{g}^{*} \simeq \mathfrak{g}$ in a $G$-equivariant way.

Now $R$ carries a $G$-invariant ring grading $R=\bigoplus_{k \geq 0} R[k](k \in \mathbb{Z})$ such that $R[\mathfrak{g}] \subset R[2]$. Then $R[0]=\mathbb{C}$. The Poisson bracket satisfies $[R[k], R[l]] \subset R[k+l-2]$, for all $k, l$ (see also [7]). Hence $R[2]$ is a finite-dimensional Lie subalgebra of $R$ under Poisson bracket. Our first main result is

\section{Theorem 1.}

(i) $R[2]$ is a semisimple Lie algebra, call it $\mathfrak{g}^{\prime}$. If $\mathfrak{g}$ is simple then $\mathfrak{g}^{\prime}$ is simple .

(ii) The condition $R[\mathfrak{g}] \subset R[2]$ determines the $G$-invariant ring grading on $R$ uniquely.

(iii) $R[2]+R[1]+R[0]$ is the unique maximal finite-dimensional Lie subalgebra of $R$ containing $R[\mathfrak{g}]$.

In Table 1 all the Lie algebras are complex simple; particularly, in (2) and (3) $n \geq 2$ and in (6) $n \geq 3$. The last column $V$ is a representation of $\mathfrak{g}$, written as a sum of its irreducible components (only fundamental dominant representations occur here). In (3) $V \oplus L=\bigwedge^{2} \mathbb{C}^{2 n}$ where $L \simeq \mathbb{C}$ is $\mathfrak{s p}(2 n)$-invariant. In (8) $V$ is the sum of the standard and the two half-spin representations. 
TABLE 1

\begin{tabular}{llll} 
& $\mathfrak{g}$ & $\mathfrak{g}^{\prime}$ & $\mathrm{V}$ \\
\hline$(1)$ & $G_{2}$ & $\mathfrak{s o}(7)$ & $\mathbb{C}^{7}$ \\
$(2)$ & $\mathfrak{s o}(2 n+1)$ & $\mathfrak{s o}(2 n+2)$ & $\mathbb{C}^{2 n+1}$ \\
$(3)$ & $\mathfrak{s p}(2 n)$ & $\mathfrak{s l}(2 n)$ & $\left(\wedge^{2} \mathbb{C}^{2 n}\right) / \mathbb{C}$ \\
$(4)$ & $F_{4}$ & $E_{6}$ & $\mathbb{C}^{26}$ \\
$(5)$ & $\mathfrak{s l}(3)$ & $G_{2}$ & $\mathbb{C}^{3} \oplus \wedge^{2} \mathbb{C}^{3}$ \\
$(6)$ & $\mathfrak{s o}(2 n)$ & $\mathfrak{s o}(2 n+1)$ & $\mathbb{C}^{2 n}$ \\
$(7)$ & $\mathfrak{s o}(9)$ & $F_{4}$ & $\mathbb{C}^{16}$ \\
$(8)$ & $\mathfrak{s o}(8)$ & $F_{4}$ & $\mathbb{C}^{8} \oplus \mathbb{C}^{8} \oplus \mathbb{C}^{8}$ \\
$(9)$ & $G_{2}$ & $\mathfrak{s o}(8)$ & $2 \mathbb{C}^{7}$
\end{tabular}

Theorem 2. Table 1 gives a complete list of the simple Lie algebra pairs $\left(\mathfrak{g} \subset \mathfrak{g}^{\prime}\right)$ that arise in Theorem 1 with $\mathfrak{g} \neq \mathfrak{g}^{\prime}$. Furthermore $\mathfrak{g}^{\prime}=\mathfrak{g} \oplus V$ as $\mathfrak{g}$-modules.

Now the Poisson bracket on $R$ defines an alternating bilinear form $\beta$ on $R[1]$ and a Lie algebra homomorphism $R[2] \rightarrow \mathfrak{s p} \beta$.

Theorem 3. $\beta$ is a symplectic form on $R[1]$ so that $R[1] \oplus R[0]$ is a Heisenberg Lie algebra. If $\mathfrak{g}$ is simple then $R[1] \neq 0$ in one and only one case, namely, when $\mathfrak{g}$ is of type $C_{n}$ for some $n$ and $M$ is the simply connected (double covering) of the minimal nontrivial nilpotent orbit of $\mathfrak{g}$. In that case $R[2] \simeq \mathfrak{s p}(2 n)$ and $R[1] \simeq \mathbb{C}^{2 n}$ generates $R$ freely.

Now the functions in $\mathfrak{g}^{\prime} \simeq R[2]$ define a map $\phi: M \longrightarrow \mathfrak{g}^{\prime}$ (again identify $\left(\mathfrak{g}^{\prime}\right)^{*} \simeq$ $\left.\mathfrak{g}^{\prime}\right)$. Let $G^{\prime}$ be the simply connected Lie group with Lie algebra $\mathfrak{g}^{\prime}$. The next result says that up to birationality $\phi$ is a moment map for $G^{\prime}$.

Theorem 4. The image $\phi(M)$ lies in a nilpotent orbit $O^{\prime}$ of $G^{\prime}$ and $\phi(M)$ is Zariski open dense in $O^{\prime}$. There exists a unique $G^{\prime}$-covering $\nu^{\prime}: M^{\prime} \longrightarrow O^{\prime}$ such that $M^{\prime}$ contains $M$ and $\nu^{\prime}$ extends $\phi$. Moreover $M$ and $M^{\prime}$ have the same regular functions, that is, $R(M)=R\left(M^{\prime}\right)$, and also the same fundamental groups, that is, $\pi_{1}(M) \simeq \pi_{1}\left(M^{\prime}\right)$.

We can construct $M^{\prime}$ in the following way. Given $M$, let $X=\operatorname{Spec} R$ be the maximal ideal spectrum of the finitely generated $\mathbb{C}$-algebra $R$. Then $X$ is a normal affine variety. Furthermore $X$ contains $M$ as an open dense subset. We call $X$ the normal closure of $M$. Indeed if $M=O$ and $\bar{O}$ is normal then $X=\bar{O}$.

Our construction is: $G^{\prime}$ acts on $X$ and $M^{\prime}$ is the unique Zariski open orbit of $G^{\prime}$ on $X$. Note that $M$ is the unique Zariski open orbit of $G$ on $X$ so that clearly $M \subset M^{\prime} \subset X$.

Thus the pair $\left(M \subset M^{\prime}\right)$ constitutes an orbit (cover) "shared" between $G$ and $G^{\prime}$. Moreover $M$ and $M^{\prime}$ have the same normal closure so that $X$ is exactly shared between $G$ and $G^{\prime}$.

Now even though $X$ may be singular we will say an isomorphism of $X$ is symplectic if the corresponding automorphism of $R$ preserves the Poisson bracket structure.

Theorem 5. Assume $R[1]=0$. Then $X$ is a singular variety. The action of any connected Lie group of holomorphic symplectic isomorphisms of $X$ that extends the action of $G$ is given by a subgroup of $G^{\prime}$. 
Hence, assuming $\mathfrak{g}$ is simple, the example of Theorem 3 is the one and only one choice of $M$ such that $X$ is smooth, and in that case one has $X \simeq \mathbb{C}^{2 n}$.

\section{Explanation of the table}

We now describe for each of the nine cases in Table 1 a choice of $M$ such that $(M, \mathfrak{g})$ gives rise to $\left(M^{\prime}, \mathfrak{g}^{\prime}\right)$. In each case $M^{\prime}=O^{\prime}$ is the orbit of the highest root vector in the simple Lie algebra $\mathfrak{g}^{\prime}$, this is the minimal nontrivial nilpotent orbit (cf. the next section).

In cases (1)-(4) $\mathfrak{g}$ is any one of the four simple Lie algebras that are doubly laced (i.e., having two root lengths). Choose $O$ to be the orbit of a short root vector and let $M$ be its simply connected cover. Then $M=O$ in (1) while $M$ is a two-fold cover of $O$ in (2)-(4). Then $\mathfrak{g}^{\prime}$ is simply laced . Furthermore $V=V_{\alpha}$ is the irreducible $\mathfrak{g}$-representation with highest weight $\alpha$ equal to the highest short root of $\mathfrak{g}$. Case (1) is a restatement in our language of a result proved by Levasseur and Smith [4] in answer to a conjecture of Vogan [6] (see introduction).

In (5) choose $O$ to be the six-dimensional (maximal) nilpotent orbit of all principal nilpotent elements and let $M$ be the three-fold simply connected covering space of $O$. This case was discovered by us in collaboration with Vogan. A noncommutative analog of this example is given in a result of Zahid [8] .

In (6) choose $O$ to be the nilpotent orbit of Jordan type (see e.g., [3]) $\left(3,1^{2 n-3}\right)$ and let $M$ be the simply connected double cover of $O$. In (7) choose $O$ to be of Jordan type $\left(2^{4}, 1\right)$ and let $M$ be the simply connected (double cover) of $O$. In (8) choose $O$ to be of Jordan type $\left(3,2^{2}, 1\right)$ and let $M$ be the simply connected (four-fold) cover of $O$.

In (9) choose $O$ to be the unique ten-dimensional nilpotent orbit and let $M$ be the simply connected six-fold cover of $O\left(\pi_{1}(O) \simeq S_{3}\right)$. In this example Levasseur and Smith already showed in [4] that $G$ has an open dense orbit on $O^{\prime}$, again in response to a question of Vogan. Moreover Vogan has constructed a unipotent representation theoretic analogue of the example. If $\pi$ denotes the minimal unitary representation of $\mathrm{SO}(4,4)$ (see e.g., [2]) then $\pi$ extends to the outer automorphism group $A$ of $\mathfrak{g}$ and in particular to a group $S \simeq S_{3}$ that induces $A$. Vogan shows that a split form $G_{o}$ of $G_{2}$ and $S$ behave like a Howe pair with respect to $\pi$ and that $\pi \mid G_{O}$ decomposes into six irreducible components. Furthermore McGovern in [5, Theorem 4.1] has constructed a Dixmier algebra analogue of this example

Remarkably three of the four nilpotent orbits of $G_{2}$ have now appeared as "shared" orbits (the principal orbit does not appear).

Cases (5)-(8) are precisely the the pairs $\left(\mathfrak{g} \subset \mathfrak{g}^{\prime}\right)$ in the table with rank $\mathfrak{g}$ $=$ rank $\mathfrak{g}^{\prime}$. Each pair $\left(\mathfrak{g} \subset \mathfrak{g}^{\prime}\right)$ is of the form $\left(\mathfrak{s}_{0} \subset \mathfrak{s}\right)$ where $\mathfrak{s}$ is a doubly laced simple Lie algebra and $\mathfrak{s}_{0}$ is a subalgebra of $\mathfrak{s}$ containing a Cartan subalgebra of $\mathfrak{s}$ and all associated long root vectors. Moreover every such pair $\left(\mathfrak{s}_{0} \subset \mathfrak{s}\right)$ where $\mathfrak{s}_{0}$ is simple arises in $(5)-(8)$. On the other hand the pairs $\left(\mathfrak{s}_{0} \subset \mathfrak{s}\right)$ where $\mathfrak{s}_{0}$ is nonsimple occur precisely when $\mathfrak{s} \simeq \mathfrak{s p}(2 n)$ and $\mathfrak{s}_{0} \simeq \mathfrak{s p}\left(2 n_{1}\right) \oplus \cdots \oplus \mathfrak{s p}\left(2 n_{k}\right)$ where $n_{1}+\cdots+n_{k}=n$ and $k \geq 2$. These pairs do arise in Theorem 1 (when $M$ is chosen so that in each simple component one has the example of Theorem 3) and these together with (5)-(8) exhaust all equal rank pairs $\left(\mathfrak{g} \subset \mathfrak{g}^{\prime}\right)$ arising in Theorem 1 such that $\mathfrak{g}^{\prime}$ is simple.

A general result regarding the ranks of $\mathfrak{g}$ and $\mathfrak{g}^{\prime}$ is that rank $\mathfrak{g}^{\prime}>\operatorname{rank} \mathfrak{g}$ whenever $M=O$ and $\mathfrak{g} \neq \mathfrak{g}^{\prime}$. 
Two instances of a triple of Lie algebras having a "shared" orbit can be found among these examples, namely, (a) $\mathfrak{s o}(8) \subset \mathfrak{s o}(9) \subset \mathfrak{f}_{4}$ (where $\mathfrak{f}_{4}$ is of type $F_{4}$ ) and (b) $\mathfrak{g}_{2} \subset \mathfrak{s o}(7) \subset \mathfrak{s o}(8)$. This is not unexpected by the theory since we in fact prove that if $\mathfrak{h}$ is any Lie subalgebra between $\mathfrak{g}$ and $\mathfrak{g}^{\prime}$ then $\mathfrak{h}$ is semisimple and also $\mathfrak{h}$ is simple if $\mathfrak{g}$ is simple. Moreover if $H$ is the simply connected group corresponding to $\mathfrak{h}$ then the statements made in Theorem 4 and immediately afterward for $G^{\prime}$ and $X$ apply equally well to $H$ and $X$. In particular one has a unique open $H$-orbit $M^{\mathfrak{h}}$ in $X, M \subset M^{\mathfrak{h}} \subset M^{\prime}$, and $M^{\mathfrak{h}}$ covers $H$-equivariantly a nilpotent $H$-orbit $O^{\mathfrak{h}} \subset \mathfrak{h}$. Furthermore the whole graded Poisson ring structure on $R$ arising from $M$ and $O$ is the same as would arise from $M^{\mathfrak{h}}$ and $O^{\mathfrak{h}}$. In particular the maximal semisimple Lie algebra $R[2]$ remains the same.

\section{Methods of PROOF}

Two key ideas are used in proving the classification of pairs. The first is a representation theoretic. We are able to compute $\mathfrak{g}^{\prime} \simeq R[2]$ as a $\mathfrak{g}$-module (for arbitrary $\mathfrak{g}$ and $M)$. Let $\varepsilon$ be a point of $M$ lying over $e$ and let $(h, e, f)$ be a standard basis of an $\mathfrak{s l}(2)$ subalgebra $\mathfrak{a}$ of $\mathfrak{g}$. For any $\mathfrak{g}$-module $V$ let $V$ [2] be the 2-eigenspace of $h$ in the fixed space $\left(V^{*}\right)^{G^{\varepsilon}}$. Then $\mathfrak{g}^{\prime} \simeq \mathfrak{g} \oplus n_{i} V_{1} \oplus \cdots \oplus n_{s} V_{s}$ where $V_{1}, \ldots, V_{s}$ is a complete list of inequivalent simple $\mathfrak{g}$-modules, excluding components of the adjoint representation, such that $n_{i}=\operatorname{dim} V_{i}[2]$ is nonzero. This follows by recognizing that the grading on $R$ comes from exponentiating a natural action of a Cartan subalgebra in $\mathfrak{a}$.

The second idea is due to David Vogan who observed that for a given pair $\left(\mathfrak{g}, \mathfrak{g}^{\prime}\right)$ arising in Theorem 1 we may change (if necessary) the choice of $O$ and $M$ so that $O^{\prime}$ is minimal . Vogan himself has determined the pairs $\left(\mathfrak{g}, \mathfrak{g}^{\prime}\right)$ in many of the cases listed above.

A principle used in setting up the theory is that one should study minimal embeddings of $X$ in order to study $M$ (again $M$ is arbitary). We prove the covering map $\nu$ extends to a finite $G$-morphism $\bar{\nu}: X \rightarrow \bar{O}$ and then the fiber $\nu^{-1}(0)$ over zero is a single point $o$. Then $o$ is the unique $G$-fixed point in $X$ and the maximal ideal of $R$ corresponding to $o$ is $m=\bigoplus_{k=1}^{\infty} R[k]$. Furthermore, $X$ is singular if and only if $X$ is singular at $o$ and then $o$ is the "most" singular point of $X$ (cf. Theorems 3 and 5 ).

We say that the pair $(v, V)$ defines an embedding of $X$ in case $V$ is a $G$-module and $v \in V^{G^{\varepsilon}}$ (where $\varepsilon \in M$ ) is such that the natural map $M \rightarrow G \cdot v$ extends to a $G$ isomorphism $X \rightarrow \overline{G \cdot v}$. We find that the Zariski tangent space $T_{o}(X)=\left(\mathrm{m} / \mathrm{m}^{2}\right)^{*}$ at $o$ provides a minimal embedding for $X$.

Theorem 6. There exists a vector $u \in T_{o}(X)^{G^{\varepsilon}}$ such that $(\mathrm{i})\left(u, T_{o}(X)\right)$ defines an embedding of $X$ and (ii) if $(v, V)$ is any pair that defines an embedding of $X$ then there exists a surjective $G$-map $\tau: V \rightarrow T_{o}(X)$ such that $\tau(v)=u$.

Clearly one has an injection $R[2] \rightarrow m / m^{2}$ when $R[1]=0$. Regarding the normality of $\bar{O}$ we find that $\bar{O}$ is a normal variety if and only if as $G$-modules $R[\mathfrak{g}] \simeq R[2] \simeq m / m^{2}$.

The proofs of Theorems 1, 3, and 5 are all applications of the following general transitivity theorem for coadjoint orbits of a special kind of Lie algebra. This is one of our main results. 
Theorem 7. Assume that $\mathfrak{s}$ is a finite-dimensional Lie algebra over $\mathbb{R}$ or $\mathbb{C}$ and that $\mathfrak{s}$ is a semidirect sum $\mathfrak{s}=\mathfrak{r}+\mathfrak{u}$ where $\mathfrak{u}$ is an abelian ideal in $\mathfrak{s}, \mathfrak{r}$ is a semisimple Lie subalgebra of $\mathfrak{s}$, and $\mathfrak{u}^{\mathfrak{r}}=0$. One may regard $\mathfrak{s}^{*}=\mathfrak{r}^{*}+\mathfrak{u}^{*}$ in an obvious way. Let $\gamma \in \mathfrak{s}^{*}$ and write $\gamma=\mu+\lambda$ with $\mu \in \mathfrak{r}^{*}$ and $\lambda \in \mathfrak{u}^{*}$. Then one has $\mathfrak{s} \cdot \gamma=\mathfrak{r} \cdot \gamma$ if and only if $\lambda=0$.

Theorem 7 says that if $\lambda \neq 0$ then the subgroup of $\operatorname{Ad} \mathfrak{s}$ corresponding to $\mathfrak{r}$ cannot operate transitively (even infinitesmally) at $\gamma$ on the coadjoint orbit.

\section{AN APPLICATION TO SYMMETRY OF FLAG VARIETIES}

Finally we give an application of our results to a well-known problem in geometry. If $P$ is a parabolic Lie subgroup of a simple Lie group $G$ it is a solved problem (see [1]) to determine the connected component of the full group $F$ of holomorphic automorphisms of the projective variety $G / P$. It is precisely for the $G$ given in cases (1), (2), and (3) in the table that $P$ exists so that $F$ is larger than that given by the action of $G$. Furthermore in those cases $F$ is in fact given by the action of $G^{\prime}$. We obtain a stronger statement (and recover the known result) in

Theorem 8. Let $P$ be any parabolic subgroup of $G$ and choose $M$ to be the unique open orbit in $T^{*}(G / P)$ so that we can take $O$ to be the $G$-orbit in $\mathfrak{g}$ of a Richardson element in the nilradical of Lie $P$. Then one has a desingularization map $T^{*}(G / P) \rightarrow X$ and the pullback of $R$ is the full ring of regular functions on $T^{*}(G / P)$. Furthermore the action of $G^{\prime}$ on $X$ lifts uniquely to an action as a group of symplectic holomorphic automorphisms of $T^{*}(G / P)$ and as such $G^{\prime}$ is maximal. $G^{\prime}$ preserves the cotangent polarization of $T^{*}(G / P)$ so that $G^{\prime}$ acts on $G / P$ and hence there exists a parabolic subgroup $P^{\prime} \subset G^{\prime}$ such that $G / P=G^{\prime} / P^{\prime}$. The action of $G^{\prime}$ on $G / P$ is the connected component of the group of all holomorphic automorphisms of $G / P$. Consequently any connected Lie group of symplectic holomorphic automorphisms of $T^{*}(G / P)$ containing the action of $G$ automatically preserves the cotangent space polarization of $T^{*}(G / P)$ and consequently will act as a group of holomorphic automorphisms of $G / P$.

\section{ACKNOWLEDGMENTS}

The authors thank Madhav Nori and David Vogan for helpful discussions. The

first author thanks the Laboratoire Mathématiques Fondamentales of Université Paris VI for its hospitality.

\section{REFERENCES}

1. M. Demazure, Automorphismes et déformations des variétés de Borel, Invent. Math. 39 (1977), 179-186.

2. B. Kostant, The vanishing of scalar curvature and the minimal representation of $S O(4,4)$, Operator Algebras, Unitary Representations, Enveloping Algebras, and Invariant Theory (A. Connes, et al, eds.), Birkhäuser, Boston, MA, 1990, pp. 85-124.

3. H. Kraft and C. Procesi, On the geometry of conjugacy classes in classical groups, Comment. Math. Helv. 57 (1982), 539-602.

4. T. Levasseur and S. P. Smith, Primitive ideals and nilpotent orbits in type $G_{2}$, J. Algebra 114 (1988), 81-105.

5. W. M. McGovern, Dixmier algebras and the orbit method, Operator Algebras, Unitary Representations, Enveloping Algebras, and Invariant Theory (A. Connes, et al, eds.), Birkhäuser, Boston, MA, 1990, pp. 397-416. 
6. D. A. Vogan, The orbit method and primitive ideals for semisimple Lie algebras, Lie Algebras and Related Topics, CMS Conf. Proc., vol. 5, Amer. Math. Soc., Providence, RI, 1986, pp. 281-316.

7. - Noncommutative algebras and unitary representations, Proc. Sympos. Pure Math., vol. 48, Amer. Math. Soc., Providence, RI, 1988 pp. 35-60.

8. A. Zahid,, Les endomorphismes $\mathfrak{k}$-finis des modules de Whittaker, Bull. Soc. Math. France 117 (1989), 451-477.

Department of Mathematics, Pennsylvania State University, University Park, Pennsylvania 16802

Department of Mathematics, Massachusetts Institute of Technology, Cambridge, Massachusetts 02139 